\title{
PERIODIC VARIATIONS AND MASS LOSS IN Be STARS
}

\author{
HIDEYUKI SAIO \\ Astronomical Institute, Faculty of Science, Tohoku University, Sendai 980, Japan
}

\begin{abstract}
We discuss the connection between the periodic light variations and the equatorial mass loss of Be stars. The observed properties of the short period ( $\sim$ day) variations seem to indicate that they arise in the photosphere. An upper limit for the surface magnetic field of $\mathrm{Be}$ stars is derived from the rate of angular momentum loss expected from the typical mass loss-rate in Be stars. The upper limit suggests that surface magnetic fields of Be stars are too weak to make a spot. We argue that the periodic variations of $\mathrm{Be}$ stars are explained by nonradial pulsations whose periods on the stellar surface are much longer than the rotation period. They transport angular momentum from the core to the envelope to accelerate the surface regions. If this mechanism works sufficiently well, the rotation speed near the surface will reach to the critical velocity and an excretion disk will be formed around the star. A simple model for a steady-state excretion disk around a $\mathrm{Be}$ star is found to be consistent with the density structure inferred from the IR fluxes.
\end{abstract}

\section{Introduction}

Be stars are B-type stars which have or had emission in the Balmer lines (see e.g., Slettebak 1988, Bjorkman \& Cassinelli 1993 for recent reviews). The emission line profile and polarization of light are consistent with the existence of an equatorial disk around a Be star.

Be stars, which tend to have large projected rotational velocities, show various light and spectral variations with timescales ranging from a fraction of a day to years. Among the variations, many Be stars show short period ( day) light variations (e.g., Balona 1990), which seem to be associated with low-order line profile (asymmetry) variations (e.g., Baade 1987). According to Balona (1990), we call these stars $\lambda$ Eri stars.

Another important property of Be stars is that the mass-loss rate determined from the IR excess is much higher than the rate from UV resonance lines, which is interpreted as that the mass-loss rate is much higher in the equatorial disk compared to the radiation driven wind in the polar region (Waters et al. 1987).

In this paper we regard rapid rotation and short-period light (and loworder line profile) variation as necessary conditions for a star to become a $\mathrm{Be}$ star, and discuss the relation between the short-period variation and mass loss.

\section{Observed Properties of the Periodic Variations of Be Stars}

Fig. 1 shows the position of $\lambda$ Eri stars on the HR diagram together with $\beta$ Cep stars (Lesh \& Aizenman 1978) and slowly pulsating B (SPB) stars (Waelkens 1991; sometimes called 53 Per stars). As seen in this figure, $\lambda$ Eri 


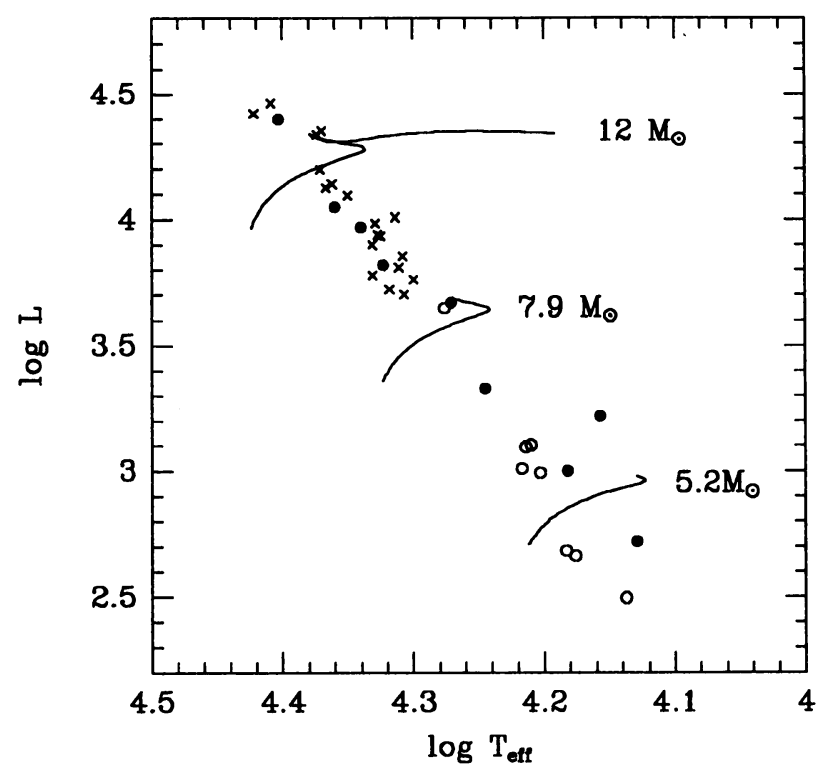

Fig. 1. Loci of some $\lambda$ Eri stars (filled circles) on the HR diagram with some $\beta$ Cep stars (crosses) and slowly pulsating $B$ stars (open circles).

stars are located in a large area of the main-sequence band covering the $\beta$ Cep and the SPB regions. However, there are clear distinctions between $\lambda$ Eri stars and the latter two groups. Firstly, there are many $\lambda$ Eri stars in SMC and LMC clusters, while no $\beta$ Cep or SPB stars are found in these clusters (Balona 1992; 1993). Pulsations of $\beta$ Cep and SPB stars are known to be excited by the kappa mechanism at the opacity peak caused by the ionization of Fe at $T \sim 2 \times 10^{5} \mathrm{~K}$ (Kiriakidis et al. 1992, Moskalik \& Dziembowski 1992, Dziembowski \& Pamyatnykh 1993, Gautschy \& Saio 1993). The absence of these variables in LMC and SMC clusters indicates that the kappa mechanism is too weak because of the low metallicity of the SMC and LMC. (Waelkens et al., 1991, found observational evidence for the metallicity dependence of the $\beta$ Cep instability strip.)

Another distinction can be seen in the period-luminosity relation. Fig. 2 shows the period-luminosity relation for some $\lambda$ Eri stars as well as for $\beta$ Cep stars and SPB stars. If pulsations are excited by the kappa mechanism at an opacity peak, the periods should be comparable to the thermal timescale of the zone above the opacity peak (Cox 1974). This relation may be converted to $\Pi \propto L^{-0.9}$ for massive main sequence stars (cf. Gautschy \& Saio 1993), where $\Pi$ and $L$ stand for the period of pulsation and the stellar luminosity, respectively. It is clearly seen in Fig. 2 that this relation connects the $\beta$ Cep region with the region of SPB stars. This indicates that these variables have a common cause of excitation. The period-luminosity relation for $\lambda$ Eri stars are different from the other stars. These facts suggest that the periodic light 


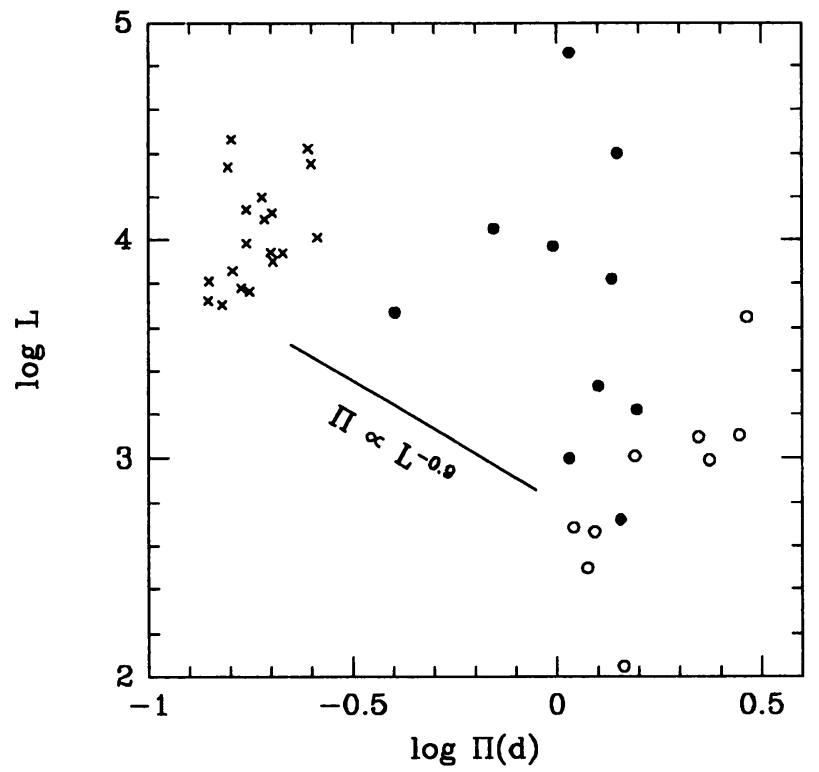

Fig. 2. Period-luminosity relation of $\lambda$ Eri stars (filled circles) with some $\beta$ Cep stars (crosses) and slowly pulsating B stars (open circles). The straight line indicates the gradient with which the kappa mechanism at the Fe peak works most effectively.

variations of Be stars are different from nonradial pulsations excited by the kappa mechanism at the Fe opacity peak.

Balona (1990) found that the periods of $\lambda$ Eri stars correlate strongly with the projected rotation velocity $v \sin i$. This correlation indicates that stellar rotation plays an important role in the periodic variations. Possible causes of the light variations are spots on the stellar surface, an inhomogeneous circumstellar envelope or nonradial pulsations whose period in the co-rotating frame is much longer than the rotation period (\$4).

Baade $(1982,1984)$ found that low-order line profile variations (changing asymmetry) are accompanied by changes in radial velocity at absorption line minima. Such radial-velocity variations do not necessarily mean the existence of a motion with this velocity on the stellar surface. Since absorption lines are rotationally broadened, radial velocity variations at absorption minima can be produced merely by a change in the apparent longitude of the temperature inhomogeneity due to rotation (e.g. Lee et al. 1992).

Another important property of the short period light variations is that there is no correlation between $\mathrm{H} \alpha$ emission and the amplitude of photometric variations (Balona et al. 1991). This property indicates that the periodic variations originate in the photosphere and not in the circumstellar envelope.

Occasional sudden changes in the light curve on a timescale of a day pose difficulties in our understanding of the phenomena of $\lambda$ Eri stars. Stagg et al. (1988) observed an abrupt increase in the amplitude of the light vari- 


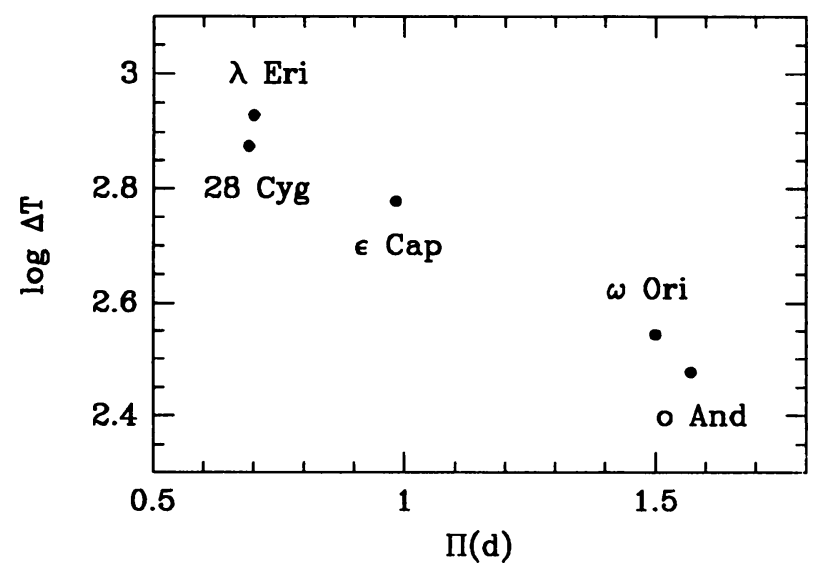

Fig. 3. The amplitude of temperature variation derived from FUV-optical observation (Percy \& Peters 1991) versus period.

ation in EW Lac. Since this phenomenon was accompanied by an abrupt change in the phase of its periodic variation, it is likely that the persistent periodic light variation was interrupted by another phenomenon. This phenomenon is probably similar to the sudden appearance of periodic variations accompanied by a large increase in the mean brightness observed in the $\mathrm{Be}$ star $\kappa$ CMa by Balona (1990). These phenomena could be related to the transition phases of $\mu$ Cen observed by Hanuschik et al. (1993), in which a circumstellar disk is being formed. In such transition phases periodic light variation can be produced by the existence of inhomogeneities in the newlyborn circumstellar envelope. (Hanuschik et al., 1993, found that such an inhomogeneity was erased in a few revolutions.) In the remainder of this paper, we will consider persistent periodic variations only.

In Fig. 3 the amplitude of the photospheric temperature variation $\Delta T$ determined from FUV-optical data (Percy \& Peters 1991, Peters 1991) is plotted against the period of variation. It is apparent that the temperature variation is larger for stars with shorter periods. Although the number of stars for which $\Delta T$ is determined is still small, if this correlation is confirmed by other $\lambda$ Eri stars, it would give an important constraint for the model of the light variations.

We consider in the following sections two possibilities for the photospheric variations which cause light and low-order line profile variations in $\lambda$ Eri stars. One is the photospheric temperature inhomogeneity (spots) possibly generated by a dipole magnetic field. The other possibility is a nonradial pulsation which has a low azimuthal degree $(m)$ and a small oscillation frequency in the co-rotating frame of the envelope. 


\section{Magnetic Fields}

A strong magnetic field could make spots on the photosphere, but such a field has not been detected in $\lambda$ Eri stars. The observationally estimated upper limit of the surface magnetic field is $\sim 10^{2}$ gauss (Baker 1987, Bohlender, these Proceedings). Smith \& Polidan (1993) inferred the existence of $\sim 10^{2}$ gauss magnetic fields in $\lambda$ Eri from its peculiar line profile variations.

An upper limit of the surface magnetic field may be estimated theoretically in a way similar to that employed by Washimi et al. (1993). The total angular momentum $\mathcal{J}$ of a uniformly rotating star may be written as

$$
\mathcal{J}=f M R^{2} \Omega,
$$

where $M, R$, and $\Omega$ are the mass, radius, and the angular frequency of rotation respectively and $f \sim 0.1$ for a massive main-sequence star (e.g., Eriguchi et al. 1992). The rate of angular momentum loss from a magnetic star $\mathcal{J}$ is estimated as

$$
\dot{\mathcal{J}} \simeq \dot{M} \Omega R_{A}^{2},
$$

where $\dot{M}$ and $R_{A}$ are mass loss rate and the Alfven radius respectively. Since Be stars have no preferred evolutionary phase in the main-sequence band (see e.g., Mermilliod 1982, Grebel et al. 1992), the timescale of spin down $(\mathcal{J} / \mathcal{J})$ should be longer than the main-sequence life time $\tau_{M S}$. This condition may be written as

$$
f \frac{M}{\dot{M}}\left(\frac{R}{R_{A}}\right)^{2}>\tau_{M S}
$$

The relation between the strength of a dipole magnetic field on the surface $B_{0}$ and at the Alfven radius gives the following relation

$$
\left(\frac{R_{A}}{R}\right)^{2}=\left(\frac{B_{0}}{2}\right)^{2 / 3}\left(4 \pi \rho_{A} v_{A}^{2}\right)^{-1 / 3},
$$

where $\rho_{A}$ and $v_{A}$ are, respectively, the gas density and outward velocity at the Alfven radius. Substituting Eq. 3.4 into inequality 3.3, we obtain a constraint on $B_{0}$;

$$
B_{0}<4 \sqrt{\pi \rho_{A}} v_{A}\left(f \frac{M}{\dot{M} \tau_{M S}}\right)^{3 / 2} .
$$

If we adopt the following typical values $\rho_{A} \sim 10^{-13} \mathrm{~g} \mathrm{~cm}^{-3}, v_{A} \sim 20 \mathrm{~km} \mathrm{~s}^{-1}$, $M / \dot{M} \sim 10^{9} \mathrm{yr}$, and $\tau_{M S} \sim 10^{7} \mathrm{yr}$, we have a constraint of $B_{0} \lesssim 100$ gauss, which is consistent with the observationally determined upper limit.

In order for a spot to be formed on the photosphere, the magnetic pressure should be comparable to (or larger than) the photospheric pressure. The upper limits of surface magnetic pressure corresponding to the upper limit of 
$B_{0}$ is $\sim 500$ dyn $\mathrm{cm}^{-2}$. On the other hand, photospheric pressure may be estimated from the equation of the hydrostatic equilibrium as $P_{\mathrm{ph}} \sim g / \kappa \sim 10^{4}$ dyn $\mathrm{cm}^{-2}$, where $g$ is the surface gravity and $\kappa$ opacity. Apparently, the pressure is much larger than the upper limit of the magnetic pressure at the photosphere. Therefore, it is difficult to make a spot on the photosphere of a Be star. This conclusion agrees with the fact that the observed correlation between emission line widths and $v \sin i$ is consistent with an axially symmetric equatorial disk (Slettebak et al. 1992 and references therein). If strong magnetic fields exist in Be stars, the distribution of gas in the disk would be non-axisymmetric as it is in the disk of the magnetic B star $\sigma$ Ori E (e.g., Bolton et al. 1987).

\section{Nonradial Pulsation}

If no strong global magnetic field exists on the surface of $\lambda$ Eri stars, it is natural to consider that nonradial pulsations cause the periodic light variations of these stars.

The relation between the angular frequency of a nonradial oscillation in the co-rotating frame, $\omega$, and in the observer's frame, $\sigma$, is $\sigma=\omega-m \Omega$, where $\Omega$ is the angular frequency of rotation. If $\omega$ is much smaller than $|m| \Omega$, $\sigma$ should be close to $|m| \Omega$ and the period in the observers frame should be close to $\Pi_{\text {rot }} /|m|$, where $\Pi_{\text {rot }}$ is the rotation period. Therefore, if very slow $(\omega \ll \Omega)$ oscillations with low azimuthal degree $(m)$ are excited, they can produce light variations and low-order line profile variations as seen in $\lambda$ Eri stars. If modes with $m=-1$ and -2 are excited simultaneously, a doublewave light curve can be produced.

Lee \& Saio $(1986,1987)$ have suggested that such low-frequency nonradial pulsations are excited in a rotating massive star, in which an oscillatory convective mode in the convective core couples with a high order $g$-mode in the envelope to form an overstable convective mode. Lee (1988) found that the same mechanism also works in a differentially rotating star. In this case the observed period should be close to the rotation period of the core divided by $|m|$. If the differential rotation in $\lambda$ Eri stars is weak, the observed periods should correlate with the rotational velocity as actually found by Balona (1990).

The modes excited by this mechanism are prograde in the co-rotating frame of the core. Since it is likely that rotation in the core is faster than in the envelope, the nonradial pulsations are likely to be prograde in the envelope. As we discuss in the following section (see also Lee \& Saio 1993), a prograde nonradial pulsation transports angular momentum from the core to the the surface region. 


\section{Angular Momentum Transport by Nonradial Pulsations}

If a non-axisymmetric nonradial pulsation is excited in a rotating star, it transports angular momentum. Ando (1986) argues that this effect may be the cause of the periodic appearance of the Be phenomenon in B-type stars. This section presents simple arguments based on Osaki (1986) concerning the transport of angular momentum by nonradial pulsations. For simplicity, we neglect the effect of the Coriolis force and consider only the equatorial plane in the following discussion. In the co-rotating frame, the flux of angular momentum is $r \rho\left\langle v_{\phi} v_{r}\right\rangle$, where $v_{r}$ and $v_{\phi}$ are, respectively, the radial and azimuthal velocities caused by the nonradial pulsation and $\langle\ldots\rangle$ means the average over one period of pulsation. In our present simplified treatment, the velocity of pulsation is given as $v=\partial \zeta / \partial t$ with the displacement vector of pulsation $\zeta$, which is written as (e.g., Unno et al. 1989)

$$
\zeta \simeq \operatorname{Re}\left\{e^{i \omega t}\left[\xi_{r}(r) \mathbf{e}_{r}+\xi_{h}(r)\left(\mathbf{e}_{\theta} \frac{\partial}{\partial \theta}+\mathbf{e}_{\phi} \frac{1}{\sin \theta} \frac{\partial}{\partial \phi}\right)\right] Y_{l}^{m}(\theta, \phi)\right\},
$$

where $Y_{l}^{m}(\theta, \phi) \propto \exp (i m \phi)$ is spherical harmonics, $\xi_{r}$ and $\xi_{h}$ are the amplitudes in the radial and horizontal directions, $\mathbf{e}_{r}, \mathbf{e}_{\theta}$, and $\mathbf{e}_{\phi}$ are the unit vectors in the $r-, \theta$, and $\phi$-directions, respectively, and $\operatorname{Re}(. .$.$) means the$ real part of the indicated quantity. From Eq. 5.1, the velocity components $v_{r}$ and $v_{\phi}$ reduce to

$$
v_{r}=C_{l m} \operatorname{Re}\left(i \omega \xi_{r} e^{i(m \phi+\omega t)}\right), \quad v_{\phi}=C_{l m} \operatorname{Re}\left(-m \omega \xi_{h} e^{i(m \phi+\omega t)}\right),
$$

where $C_{l m}$ is a normalization constant.

In the adiabatic approximation, $\xi_{r}, \xi_{h}$ and $\omega$ are real and hence the velocity components $v_{r}$ and $v_{\phi}$ may be written as

$$
v_{r}^{\text {ad }}=A \sin (\omega t+m \phi), \quad v_{\phi}^{\text {ad }}=A k m \cos (\omega t+m \phi),
$$

where $A=-\xi_{r} C_{l m} \omega$ and $k=\xi_{h} / \xi_{r}$. Note that the phases of $v_{r}^{\text {ad }}$ and $v_{\phi}^{\text {ad }}$ differ by $\pi / 2$ and hence $\left\langle v_{r} v_{\phi}\right\rangle=0$ (i.e., no angular momentum flux) in this case.

For nonadiabatic pulsations $\xi_{r}, \xi_{h}$, and $\omega$ are complex. To make our analysis simple, we disregard the imaginary part of $\omega$. Then the velocity components $v_{r}$ and $v_{\phi}$ may be written as

$$
v_{r}=A \sin (\omega t+m \phi), \quad v_{\phi}=A m k \cos (\omega t+m \phi+\delta) .
$$

The phase difference between $v_{r}$ and $v_{\phi}$ deviates from $\pi / 2$ by $\delta$. In this case $\left\langle v_{r} v_{\phi}\right\rangle$ has a finite value and the angular momentum flux $\mathcal{F}_{J}$ is given as

$$
\mathcal{F}_{J}=\frac{\omega r \rho}{2 \pi} \int_{0}^{2 \pi / \omega} v_{\phi} v_{r} d t=-\frac{1}{2} m A^{2} k r \rho \sin \delta
$$


Next we consider the relation between $\mathcal{F}_{J}$ and the energy flux of the nonradial pulsation $\mathcal{F}_{E}$. The energy flux may be evaluated as the rate of work done by the nonradial pulsation: i.e.,

$$
\mathcal{F}_{E}=\left\langle v_{r} p^{\prime}>=\frac{\omega}{2 \pi} \int_{0}^{2 \pi / \omega} v_{r} p^{\prime} d t\right.
$$

where $p^{\prime}$ is the Eulerian perturbation of pressure. The pressure perturbation is related to the pulsation velocity through the momentum equation in the $\phi$ direction (on the equatorial plane) by

$$
-\frac{1}{\rho r} \frac{\partial p^{\prime}}{\partial \phi} \simeq \partial v_{\phi} / \partial t=-m A k \omega \sin (\omega t+m \phi+\delta) .
$$

Integrating this equation with respect to $\phi$, we obtain

$$
p^{\prime} \simeq-A k \omega \rho r \cos (\omega t+m \phi+\delta)=-v_{\phi} \omega \rho r / m .
$$

Substituting Eq. 5.8 into Eq. 5.6 and using Eq. 5.5, we obtain the relation between $\mathcal{F}_{E}$ and $\mathcal{F}_{J}$,

$$
\mathcal{F}_{E}=-\frac{\omega^{2} \rho r}{2 \pi m} \int_{0}^{\frac{2 \pi}{\omega}} v_{r} v_{\phi} d t=-\omega \mathcal{F}_{J} / m=\frac{1}{2} \omega r \rho A^{2} k \sin \delta .
$$

It is apparent that a prograde mode $(\omega / m<0)$ transports angular momentum in the same direction as the energy flow.

The low-frequency nonradial pulsations which are overstable in a rotating massive main-sequence star are likely to be prograde (see $\$ 4$ ). For these oscillations energy flows from the convective core to the damping zone near the surface (Lee \& Saio 1989). Therefore angular momentum is transported outward and the surface region is accelerated. Although the photospheric rotation speeds of $\mathrm{Be}$ stars are constrained observationally to be less than 90 per cent of the critical velocities (Collins \& Sonneborn 1977), there is some indication that the rotational velocity increases outward above the photosphere (Chen et al. 1989). Therefore it is possible that the surface region is accelerated by nonradial pulsations and that the rotational velocity reaches the critical velocity at a layer above the photosphere.

\section{Excretion Disk around a Be Star}

If angular momentum is supplied steadily at the surface of a Be star, an excretion disk is formed around the star. In the excretion disk, the gravitational force in the radial direction is balanced by the centrifugal force of the Keplerian motion as in an accretion disk, but the direction of the drift is outward. Hanuschik et al. (1993) call it a decretion disk. Lee et al. (1991) 
investigated the vertical structure of excretion disks. Here we discuss simple properties of a steady excretion disk analytically.

In a steady state Keplerian disk, the conservation of angular momentum may be written as

$$
4 \pi \frac{d}{d r}\left(r^{2} W\right)+\dot{M} \sqrt{G M / r}=0,
$$

where $\dot{M}=2 \pi r \Sigma v_{r}$ represents the mass-loss rate (which is constant throughout the disk), $M$ is the mass of the central star, and $W$ is the vertically integrated viscous stress. In order to evaluate $W$ we use the $\alpha$ description for the viscous stress (Shakura \& Sunyaev 1973) and assume that the disk is vertically isothermal. Then we obtain

$$
W=\alpha \int_{-\infty}^{\infty} p d z=\alpha c_{s}^{2} \int_{-\infty}^{\infty} \rho d z=\alpha c_{s}^{2} \Sigma
$$

where $c_{s}(r)=\sqrt{p / \rho}$ is the isothermal sound speed, $\Sigma$ is the surface density, and $\alpha$ is a free parameter of $O(1)$. Integrating Eq. 6.1 yields the relation

$$
2 \pi \alpha c_{s}^{2} r^{2} \Sigma=\dot{M} \sqrt{G M r_{1}}\left(1-\sqrt{r / r_{1}}\right),
$$

where the subscript 1 indicates a quantity at the outer boundary of the disk $\left(\Sigma_{1} \sim 0\right)$. From Eq. 6.3 we obtain

$$
\Sigma \propto r^{-2} c_{s}^{-2} \text { and } v_{r}=\dot{M} /(2 \pi r \Sigma) \propto r c_{s}^{2} \quad \text { for } \quad r \ll r_{1} .
$$

In order to convert the surface density to the density on the equatorial plane, $\rho_{e}(r)$, we consider the vertical structure of the disk. Since $p=c_{s}(r)^{2} \rho$, the equation for vertical hydrostatic equilibrium, $d p / d z=-G M z \rho / r^{3}$ may be integrated to yield $\rho(z, r)=\rho_{c}(r) \exp \left[-G M z^{2} /\left(2 r^{3} c_{s}^{2}\right)\right]$. Then we have

$$
\Sigma=\int_{-\infty}^{\infty} \rho d z=\rho_{e} r^{3 / 2} c_{s} \sqrt{\frac{2 \pi}{G M}} .
$$

If we assume that the temperature of the excretion disk varies as $r^{-\beta}$ with $\beta>0$ (i.e., $c_{s} \propto r^{-\beta / 2}$ ), we obtain

$$
\rho_{e} \propto \Sigma r^{-3 / 2} c_{s}^{-1} \propto r^{-3.5+1.5 \beta} .
$$

Steady excretion models of Lee et al. (1991) indicate $\beta \sim 1-1.5$. If a shock wave is formed at the surface of the excretion disk due to ram pressure of the polar wind (Bjorkman \& Cassinelli 1993), $\beta$ would have a smaller value. In spite of our simple analysis, Eq. 6.6 is consistent with the observational relation $\rho \propto r^{-n}$ with $2<n<3.5$ obtained from IR data by Waters et al. (1987). 


\section{Acknowledgements}

I thank Shinpei Shibata and Osamu Kaburaki for helpful conversations on the effect of magnetic fields.

\section{References}

Ando, H.: 1986, Astron. Astrophys. 163, 97.

Baade, D.: 1982, Astron. Astrophys. 105, 65.

Baade, D.: 1984, Astron. Astrophys. 134, 105.

Baade, D.: 1987, in Slettebak, A. and Snow, T.P., eds., Physics of Be Stars, Cambridge Univ. Press: Cambridge, 361.

Baker: 1987, in Slettebak, A. and Snow, T.P., eds., Physics of Be Stars, Cambridge Univ. Press: Cambridge, 38.

Balona, L.A.: 1990, Mon. Not. Roy. Astr. Soc. 245, 92.

Balona, L.A.: 1992, Mon. Not. Roy. Astr. Soc. 256, 425.

Balona, L.A.: 1993, Mon. Not. Roy. Astr. Soc. 260, 795.

Balona, L.A., Sterken, C. and Manfroid, J.: 1991, Mon. Not. Roy. Astr. Soc. $252,93$.

Bjorkman, J.E. and Cassinelli, J.P.: 1993, Astrophys. J. 409, 429.

Bolton, C.T., Fullerton, A.W., Bohlender, D., Landstreet, J.D. and Gies, D.R.: 1987, in Slettebak, A. and Snow, T.P., eds., Physics of Be Stars, Cambridge Univ. Press: Cambridge, 82.

Chen, H., Riguelet, A., Sahade, J. and Kondo, Y.: 1989, Astrophys. J. 347, 1082.

Collins, G.W.,II and Sonneborn, G.H.: 1977, Astrophys. J. Suppl. 34, 41.

Cox, J.P.: 1974, Rep. Prog. Phys. 37, 563.

Dziembowski, W.A. and Pamyatnykh, A.A.: 1993, Mon. Not. Roy. Astr. Soc. 262, 204.

Eriguchi, Y., Yamaoka, H., Nomoto, K. Hashimoto, M.: 1992, Astrophys. J. 392, 243.

Gautschy, A. and Saio, H.: 1993, Mon. Not. Roy. Astr. Soc. 262, 213.

Grebel, E.K., Richtler, T. and de Boer, K.S.: 1992, Astron. Astrophys. 254, L5.

Hanuschik, R.W., Dachs, J., Baudzus, M. and Thimm, G.: 1993, Astron. Astrophys. 274, 356.

Kiriakidis, M., El Eid, M. and Glatzel, W.: 1992, Mon. Not. Roy. Astr. Soc. 255, 1 p.

Lee, U.: 1988, Mon. Not. Roy. Astr. Soc. 232, 711.

Lee, U., Jeffery, C.S. and Saio, H.: 1992, Mon. Not. Roy. Astr. Soc. 254, 185.

Lee, U. and Saio, H.: 1986, Mon. Not. Roy. Astr. Soc. 221, 365.

Lee, U. and Saio, H.: 1987, Mon. Not. Roy. Astr. Soc. 225, 643.

Lee, U. and Saio, H.: 1989, Astrophys. J. 360, 590.

Lee, U. and Saio, H.: 1993, Mon. Not. Roy. Astr. Soc. 261, 415.

Lee, U., Saio, H. and Osaki, Y.: 1991, Mon. Not. Roy. Astr. Soc. 250, 432.

Lesh, J.R. and Aizenman M.L.: 1978, Ann. Rev. Astron. Astrophys. 16, 215.

Mermilliod, J.-C.: 1982, Astron. Astrophys. 109, 48.

Moskalik, P. and Dziembowski, W.A.: 1992, Astron. Astrophys. 256, L5.

Osaki, Y.: 1986, Publ. Astr. Soc. Pacific 98, 30.

Percy, J.R. and Peters, G.J.: 1991, in Baade, D., ed., Rapid Variability of OB-stars: Nature and Diagnostic Value, ESO: Garching, 97.

Peters, G.J.: 1991, in Baade, D., ed., Rapid Variability of OB-stars: Nature and Diagnostic Value, ESO: Garching, 171.

Shakura, N.I. and Sunyaev, R.A.: 1973, Astron. Astrophys. 24, 337.

Slettebak, A.: 1988, Publ. Astr. Soc. Pacific 100, 770.

Slettebak, A., Collins, G.W. II and Truax, R.: 1992, Astrophys. J. Suppl. 81, 335.

Smith, M.A. and Polidan, R.S.: 1993, Astrophys. J. 408, 323.

Stagg, C.R., Božić, H., Fullerton, A.W., Gao, W.S., Guo, Z.H., Harmanec, P., Horn, J., Huang, L., Iliev, L.H., Koubský, P., Kovachev, B.Z., Pavlovski, K., Percy, J.R., Schmidt, F., Stefl, S., Tomov, N.A. and Žižnovský, J.: 1988, Mon. Not. Roy. Astr. 
Soc. 234, 1021.

Unno, W, Osaki, Y., Ando, Y., Saio, H. and Shibahashi, H.: 1989, Nonradial Oscillations of Stars, 2nd ed., Univ. Tokyo Press: Tokyo

Washimi, H., Mori, M. and Shibata, S.: 1993, Nature submitted.

Waters, L.B.F.M., Coté, J. and Lamers, H.J.G.L.M.: 1987, Astron. Astrophys. 185, 206.

Waelkens, C.: 1991, Astron. Astrophys. 246, 453.

Waelkens, C., Van den Abeele, K. and Van Winckel, H.: 1991, Astron. Astrophys. 251, 69.

\section{Discussion}

Smith: I have two comments to make.

(1) It may be possible to produce an excretion disk by hydrodynamical interaction of particles initially ejected from a star by a deus ex machina. In such a picture, many particles would lose angular momentum and return to the star, leaving the other particles angular-momentum rich and able to remain in orbit. Although no one has explored the validity of this model yet, it should be considered for inclusion in your list of mechanisms (even in the absence of radiation pressure).

(2) Your $J$-transfer mechanism from NRP (the Ando mechanism) relies on $m<0$. Actually, NRP models seem to always have $m>0$ and therefore the $J$-transfer will be inward, not outward! (Note that even Harmanec's lower mass-radius relation will change the sign of $m$.)

Lafon: My question is concerned with dynamics. You have a star rotating at a given (even if perturbed) velocity, but you say that the excretion disk rotates with Keplerian velocity. There are too many dynamical conditions in the star and the disk to be matched without some boundary layer between them. Can you comment about this problem in your model?

Saio: I am afraid that my model is too simple to answer your question properly. I just speculate that the rotational velocity increases outward and attains Keplerian velocity at some height above the photosphere.

Owocki: I didn't quite understand the mechanism for transporting angular momentum outward. But assuming it is true, I still don't understand why it wouldn't make the surface layers spin up past critical velocity, in contradiction to observations of widths of photospheric lines. Also, even if your angular momentum is contained in the pulsations and not simply in the systematic rotation of the atmosphere, wouldn't it still impart a centrifugal effect that would expel the pulsating photospheric material?

Saio: I speculate that the rotational velocity becomes critical above the photosphere. The perturbation of angular momentum caused by nonradial pulsations is assumed to be small, but on a timescale very long compared to the period of nonradial pulsation, angular momentum can be accumulated in the surface regions where the wave dissipates. 
Henrichs: What is the effect of Eddington-Sweet currents on the rate of outward angular momentum transport?

Saio: I did not take into account the effect of Eddington-Sweet currents, but I think that the timescale involved is much longer than the timescale of angular momentum transport by nonradial pulsation.

Hanuschik: In Hanuschik et al. (1993), we published a series of $\mathrm{H} \alpha$ observations of $\mu$ Cen (HR 5193) where we observed isolated outbursts, and a decay of emission thereafter, with a typical decay timescale of about 50-100 days. We proposed the decretion disk model in order to explain these findings. Do you have any ideas on the origin of the decay timescale and, if it is due to viscosity, about its magnitude?

Saio: The timescale of radial drift of gas in a Keplerian disk is determined by viscosity. Since we use the $\alpha$-disk assumption, the timescale depends on the parameter $\alpha$. If we assume $\alpha=1$, the drift timescale for an excretion disk around a typical Be star is a few hundred days, which is more or less consistent with your results on the decay timescale of $\mu$ Cen.

Harmanec: How can you explain the observed periodic light curves of Be stars by NRP given the fact that they are (i) singly-periodic, (ii) systematically change their amplitudes and shapes from cycle to cycle, and (iii) their periods remain constant over years?

Saio: Amplitude modulation is possible when at least two pulsation modes are excited simultaneously. If the periods of these modes are very close to each other, only one period is detected observationally unless a light curve with a very long baseline is analyzed. Since the pulsation periods are much longer than the rotation period in the co-rotating frame of the convective core, the periods in the observer's frame should be very close to the rotation period of the core divided by $|m|$ and hence should be stable. 